\title{
Post Flu Shot Confusion - think ADEM!
}

\author{
Nanjunda Murthy Jyoti Prabha MD, Sanket R. Thakore MD, Akram Zaqooq MD
}

\begin{abstract}
A 57-year-old Caucasian man with hypertension and diabetes presented with left arm weakness and headache for one day. He had an influenza vaccination 10 days prior to presentation. Computed tomography (CT) of the head showed a possible right MCA stroke. The next day he developed left hemiplegia. A repeat CT of the head exhibited a "mass like" effect in the right hemisphere. Magnetic resonance imaging (MRI) of the head showed a diffuse area of abnormal signals, involving both hemispheres, and diffuse

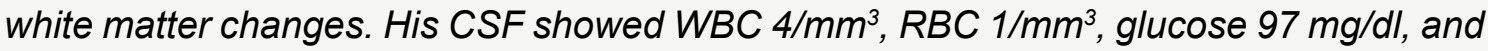
protein $96 \mathrm{mg} / \mathrm{dl}$. He continued to deteriorate, developed encephalopathy, and had to be intubated. A repeat MRI of the head demonstrated extensive bilateral, poorly marginated white matter, and some grey matter involvement characteristic of acute disseminated encephalomyelitis (ADEM). He failed to respond to methylprednisolone $1 \mathrm{gram}$ daily and was started on plasma exchange daily for 10 days. He started to improve after the second session. After five months he was essentially normal and had returned to work. This case demonstrates that routine vaccination can have important complications.
\end{abstract}

Key words: encephalitis, encephalomyelitis, influenza vaccine, demyelinating diseases

\section{INTRODUCTION}

Vaccines have a major role in preventing disease but do have side effects. Although most serious adverse effects are rare following vaccination, it is important to recognize them, as they could be potentially life threatening. Acute disseminated encephalomyelitis (ADEM), a demyelinating disease of the nervous system, is one such complication. Fewer than $5 \%$ of ADEM cases are associated with vaccinations. There is little knowledge about its pathogenesis, and treatment options are based on small case

Corresponding author: Sanket R. Thakore MD

Contact Information: Sanket.Thakore@ttuhsc.edu DOI: 10.12746/swrccc2015.0311.147 series. Here we report a 57-year-old Caucasian man who developed ADEM 10 days following an influenza vaccination. The goal of this report is to alert health care providers about such rare occurrences.

\section{CASE}

We report a 57-year-old Caucasian man with hypertension and type 2 diabetes. He was an ex-smoker but denied any history of alcohol or substance abuse and any family history of neurological disorders. He was well until one day prior to admission, when he presented with left arm weakness, slurred speech, and headache. Clinical examination and computed tomography (CT) of the head suggested acute right middle cerebral artery ischemic stroke, 
and he was admitted to a stroke unit for management. However, over the next 24 hours, he developed an altered mental status with worsening weakness in his left arm and new weakness in his left leg. On further inquiry, his family reported that the patient had received an influenza vaccination 10 days prior to his presentation.

Neurological examination on the day of admission revealed a Glasgow Coma Scale (GCS) of 15 and left arm power of 2/5. The rest of his examination was essentially normal. Laboratory results were within normal limits except for $\mathrm{HbA} 1 \mathrm{c}$ of $13 \%$. On day two, his GCS deteriorated to 12 (E4, V3, M5). Vital signs remained normal with BP $132 / 78 \mathrm{mmHg}$, temperature $97.2^{\circ} \mathrm{F}$, respiratory rate 16 breaths per minute, and heart rate 84 beats per minute. His CBC and chemistry panel were unchanged from day one. $A$ repeat $C T$ of the head showed possible mass effect in the right frontal hemisphere. Magnetic resonance imaging (MRI) of the head showed a diffuse area of abnormal signals involving both hemispheres and diffuse white matter changes. The differential diagnoses included infection, infiltrating neoplasm, and demyelinating diseases. He was started on acyclovir,

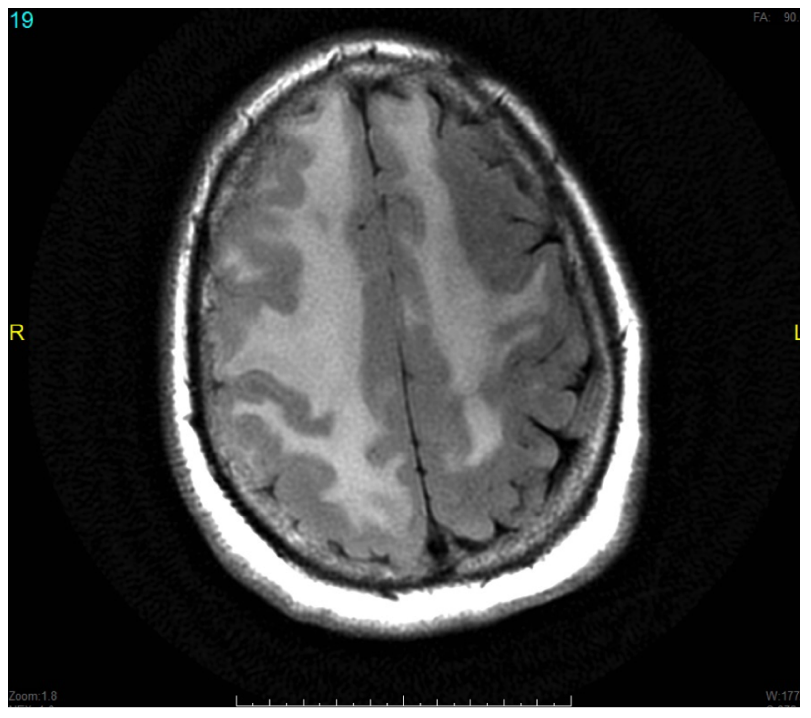

Figure1: T2 FLAIR Image. Extensive hyperintense signals on T2 FLAIR involving deep white matter of the both frontal and parietal lobes bilaterally. It exhibits multifocal, bilateral, poorly marginated white matter disease. It also shows gray matter involvement, where the cortex is very thin (red arrows in Figure 2). All lesions are about the same age. There is minimal midline shift. ceftriaxone, and vancomycin for possible meningoencephalitis.

On day three, his neurological status deteriorated. He developed spasticity in his arms and legs and had an altered mental status to a point that he needed intubation for airway protection. Opening pressure, on lumbar puncture, was normal, and CSF analysis showed a WBC count $4 / \mathrm{mm}^{3}$ (lymphocytes only), RBC $1 / \mathrm{mm}^{3}$, glucose $97 \mathrm{mg} / \mathrm{dl}$, and total protein $96 \mathrm{mg} / \mathrm{dl}$. Over the next 24 to 48 hours, the patient did not improve. A repeat MRI (Figure 1, 2) showed bilateral, poorly marginated, multifocal and extensive white matter, and some gray matter lesions of the same age. These findings suggested acute disseminated encephalomyelitis. Methylprednisolone, $1 \mathrm{gm}$ daily, was started. An extensive workup was done to rule out other diagnoses, such as HIV leading to PML, multiple sclerosis, vasculitis, Lyme disease, other viral and fungal encephalitis, and prion diseases (mainly CJD); all results were negative (Table). The CFS analysis showed increased IgG and IgG synthesis and a positive myelin basic protein, which implied an immunological process.

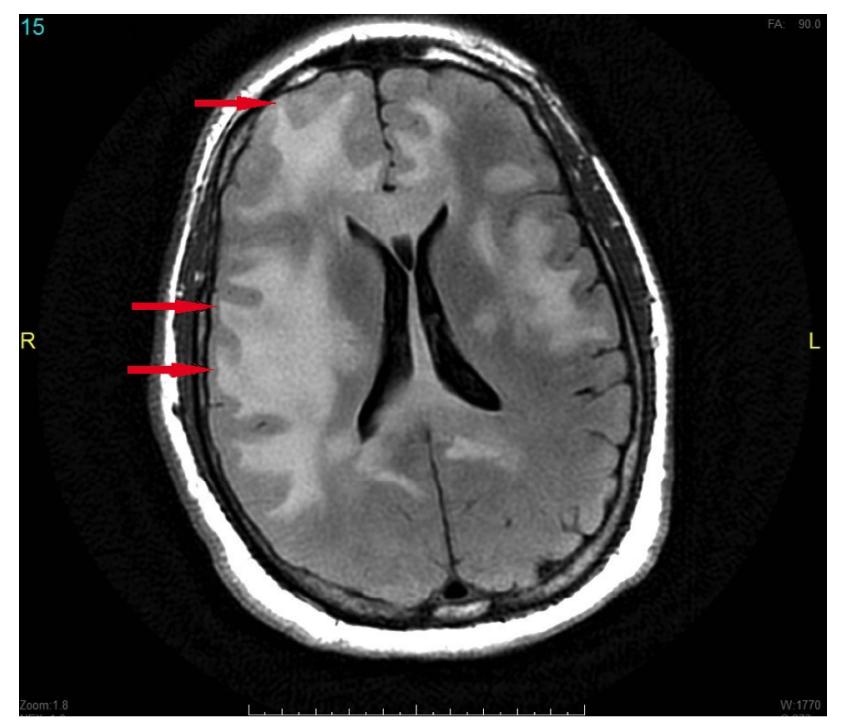

Figure2: T2 FLAIR Image. This axial image shows extensive bilateral white matter pathology of the same age. 
Table

\begin{tabular}{|l|l|l|}
\hline \multirow{4}{*}{ Serum } & Gram stain and culture & \multirow{4}{*}{ Negative } \\
\cline { 2 - 2 } & HIV by ELISA & \\
\cline { 2 - 2 } & West Nile Ig G and Ig M & \\
\cline { 2 - 2 } & Anti MPO, PR3, C-ANCA, P-ANCA, ANA & \\
\cline { 2 - 2 } & Angiotensin converting enzyme & \\
\hline CSF & Gram stain and culture & \\
\cline { 2 - 3 } & HSV 1 and 2 DNA PCR & \\
\cline { 2 - 3 } & Histoplasma M \& Y, Aspergillus, Coccidioides Abs \\
\cline { 2 - 3 } & VDRL and Lyme Abs & \\
\cline { 2 - 3 } & JC virus DNA PCR & Negative \\
\cline { 2 - 3 } & "tau protein" and "14-3-3" protein for CJD & \\
\cline { 2 - 3 } & Oligoclonal bands (none found) & \\
\hline Nasal & Influenza A \& B & \\
\hline
\end{tabular}

\section{Discussion}

Acute disseminated encephalomyelitis is an autoimmune demyelinating disease of the nervous system, commonly triggered by infection or immunization. Fewer than $5 \%$ of ADEM cases are preceded by vaccination ${ }^{1}$, and the usual onset is within 1 month post-vaccination. ${ }^{2}$ The typical presentation includes acute onset of encephalopathy (75\%), multifocal neurological findings, rapid deterioration, and a monophasic illness. ${ }^{3}$ In our case, the patient received influenza vaccine 10 days prior to presentation. If treatment is provided in a timely manner, full recovery occurs in 50 to $75 \%$ of patients within 1 to 6 months.

\section{Diagnosis}

The CSF analysis confirmed that patient had an immunological insult. The CSF results, the characteristic MRI, and clinical findings confirmed our suspicion of ADEM. He met level 1 diagnostic certainty criteria for ADEM according to Brighton Collaboration Encephalitis Working Group. ${ }^{4}$ He demonstrated multifocal neurological findings, characteristic MRI findings, and his illness was monophasic. He devel- oped encephalopathy, which is present in up to 75 $\%$ of patients with ADEM. ${ }^{5} \mathrm{He}$ had left visual neglect, left upper motor facial weakness, left hemiplegia, and increased spasticity in lower extremities. His MRI showed characteristic findings for ADEM with multifocal, bilateral, poorly marginated white matter disease with hyperintensities on T2 FLAIR image. ${ }^{1}$ It also showed gray matter involvement (red arrows in figure 2) which helps differentiate ADEM from multiple sclerosis (MS), since MS does not have gray matter involvement. All lesions of about the same age are another typical finding of ADEM on MRI, unlike MS in which older lesions appear as black holes. The third important feature of ADEM is the monophasic illness, i.e., patients should not have relapse of disease for at least for 90 days after a symptomatic nadir. Our patient was followed for five months and had no relapse of any symptoms. To prove whether ADEM in this patient was caused by the influenza vaccine or whether it was a temporal coincidence is difficult, as there is no definitive test available to prove this. According to the World Health Organization causality assessment criteria ${ }^{6}$, it was very likely that ADEM was caused by the influenza vaccine that the patient received 10 days prior to presentation. 


\section{Management}

Most treatment options for ADEM are based on case reports or on small clinical series. There are no randomized clinical trials to identify a preferred treatment. Three main treatments have shown benefit: high dose corticosteroids, plasma exchange therapy, and IV immunoglobulins. ${ }^{1,2}$ The first line therapy is high dose corticosteroids. The most commonly used regimen is methylprednisolone $1 \mathrm{gm}$ IV daily for 3 to 5 days followed by 28 days of tapering prednisone. If treatment with corticosteroids does not produce improvement, the second line therapy is plasma exchange. Most literature recommends 5 to 10 sessions of plasma exchange. Intravenous immunoglobulin is used only if plasma exchange is not available or contraindicated. In our case, the patient did not show any improvement after $1 \mathrm{gm}$ methylprednisolone IV for three days and was started on plasma exchange therapy. He received one session daily for 10 days and started improving after the second session. Within five months he had returned to work without any difficulties.

Author Affiliation: Nanjunda Murthy Jyoti Prabha and Sanket Thakore are residents in the Department of Internal Medicine at Texas Tech University Health Sciences Center in Amarillo, TX. Akram Zaqooq is an assistant professor in the Division of Critical Care Medicine at TTUHSC in Amarillo, TX.

Received: $06 / 14 / 2015$

Accepted: 06/23/2015

Reviewers: Kenneth Nugent MD

Published electronically: 07/15/2015

Conflict of Interest Disclosures: none

\section{REFERENCES}

1. Bennetto L, Scolding N. Inflammatory/post-infectious encephalomyelitis. J Neurol Neurosurg Psychiatry 2004; 75:i22-8.

2. Machicado J, Bhagya-Roa B, et al. Acute disseminated encephalomyelitis following seasonal influenza vaccination in elderly patient. Clin Vaccine Immunol 2013; 20:1485-86.

3. Shoamanesha A, Traboulsee A. Acute disseminated encephalomyelitis following influenza vaccination. Vaccine 2011; 29:818285.

4. Sejvar JJ, Kohl KS, et al. Encephalitis, myelitis, and acute disseminated encephalomyelitis (ADEM): Case definitions and guidelines for collection, analysis, and presentation of immunization safety data. Vaccine 2007; 25:5771-92.

5. Karussis D, Petrou P. The spectrum of post-vaccination inflammatory CNS demyelinating syndromes. Autoimmun Rev $2014 ; 13: 215-24$.

6. Collet JP, MacDonald N, et al. Monitoring signals for vaccine safety: the assessment of individual adverse event reports by an expert advisory committee. Bull World Health Organ 2000; 78:178-85. 